\title{
Autophagy dysfunction may be involved in the pathogenesis of ankylosing spondylitis
}

\author{
MIN TAN $^{1 *}$, QUAN-BO ZHANG ${ }^{1 *}$, TAO-HONG LIU ${ }^{1}$, YAN-YU YANG $^{1}$, \\ JIAN-XIONG ZHENG ${ }^{2}$, WEN-JUN ZHOU ${ }^{2}$, QIN XIONG ${ }^{1}$ and YU-FENG QING ${ }^{2}$ \\ Departments of ${ }^{1}$ Geriatrics and ${ }^{2}$ Rheumatology and Immunology, \\ The Affiliated Hospital of North Sichuan Medical College, Nanchong, Sichuan 637000, P.R. China
}

Received December 6, 2019; Accepted July 14, 2020

DOI: $10.3892 / \mathrm{etm} .2020 .9116$

\begin{abstract}
The present study aimed to investigate the expression and significance of the mRNA of genes associated with autophagy and long non-coding RNA (lncRNA) GAS5 in peripheral blood mononuclear cells (PBMCs) of patients with ankylosing spondylitis (AS). The mRNA levels of microtubule-associated protein light chain 3 (LC3), Beclin1, autophagy-related gene (ATG)3, ATG5, ATG12, ATG 16 ligand 1 (ATG16L1) and lncRNA growth arrest-specific 5 (GAS5) in PBMCs from 60 patients with AS and 30 healthy controls (HC) were examined by reverse transcription-quantitative PCR. The correlations between the levels of LC3, Beclin1, ATG3, ATG5, ATG12 and ATG16L1 mRNA as well as lncRNA GAS5 levels with disease activity and laboratory parameters in patients with AS were determined by Spearman correlation analysis. In addition, the diagnostic value of 1ncRNA GAS5 for AS was explored through establishing a receiver operating characteristic (ROC) curve. The results indicated that, compared to the HCs, patients with AS had lower expression levels of LC3, ATG5, ATG12, ATG16L1 and lncRNA GAS5 in their PBMCs. Compared with those in patients with inactive AS, the levels of ATG5 and ATG12 were lower than those in patients with active AS. Of note, ATG5 and ATG12 mRNA levels were negatively correlated with disease activity indexes. 1ncRNA GAS5 was positively correlated with the expression of Beclin1, ATG3,
\end{abstract}

Correspondence to: Professor Quan-Bo Zhang, Department of Geriatrics, The Affiliated Hospital of North Sichuan Medical College, 63 Wenhua Road, Nanchong, Sichuan 637000, P.R. China E-mail: quanbozhang@126.com

Professor Yu-Feng Qing, Department of Rheumatology and Immunology, The Affiliated Hospital of North Sichuan Medical College, 63 Wenhua Road, Nanchong, Sichuan 637000, P.R. China

E-mail: qingyufengqq@163.com

*Contributed equally

Key words: ankylosing spondylitis, autophagy-related genes, long non-coding RNA growth arrest-specific 5, diagnosis, disease severity and activity
ATG5, ATG12 and ATG16L1. The area under the ROC curve for the use of 1ncRNA GAS5 expression to diagnose AS was 0.808 with a $95 \%$ CI of $0.714-0.902$. In conclusion, patients with AS had decreased expression of genes associated with autophagy and lncRNA GAS5. The extent of the reduction in ATG5 and ATG12 expression levels in patients with AS was correlated with the disease severity and activity. Furthermore, lncRNA GAS5 was a diagnostic indicator of AS.

\section{Introduction}

Ankylosing spondylitis (AS) is a chronic immune-mediated type of inflammatory arthritis, mainly invading the axial skeleton, frequently accompanied by extra-articular manifestations. In severe cases, spinal deformity and ankylosis may occur. AS has a prevalence of 0.03-0.09\% and the risk of mortality and cardiovascular events, including vascular death, is increased in patients with AS (1). The aetiology and pathogenesis of AS have remained to be fully elucidated. Epidemiological investigations have indicated that genetic and environmental factors have a role in the pathogenesis of this disease. The human leukocyte antigen B27 (HLA-B27) is known to have a strong association with AS and a distinct tendency of family aggregation (2). In addition, several inflammatory pathways appear to have a relevant role in the pathogenesis of $\mathrm{AS}$, including NF- $\kappa \mathrm{B}$ and the IL-17/IL-23 pathway (3).

Autophagy is an important mechanism in which cells digest their components through lysosomes/vacuoles to maintain normal physiological activity and homeostasis. It is highly conserved from yeast to mammals. In addition to regulating cell homeostasis, autophagy also has an important role in various diseases, including cancer, neurodegeneration, cardiomyopathy and diabetes (4). Autophagy, the process by which cells degrade intracellular components, may be categorized into three major types: Macroautophagy, microautophagy and chaperonemediated autophagy (5). Macroautophagy is the major form of autophagy and has been studied most commonly; it relies on the formation of the double-membrane-bound phagophore and autophagosome. The formation of macroautophagy (hereafter referred to as 'autophagy') is divided into three stages, including the formation of pre-autophagosomes (also called isolation membranes), 
the formation of autophagosomes and the maturation of autophagy (6). Each step is regulated by different genes associated with autophagy. The core autophagy proteins, which are required for autophagosome formation, are divided into different functional subgroups: i) The autophagy-related gene (ATG)1/ULK complex; ii) ATG9 and its cycling system; iii) the PtdIns 3-kinase complex; and iv) two ubiquitin-like conjugation systems: The ATG12 [ATG5, ATG7, ATG10, ATG12 and ATG16 ligand 1 (ATG16L1)] and ATG8 (ATG3, ATG4, ATG7 and ATG8) conjugation systems (5). In addition to the interaction of autophagy proteins, long non-coding RNAs (lncRNAs) are also involved in the regulation of autophagy (7), including lncRNA autophagy-promoting factor (8), lncRNA gallbladder cancer drug resistance-associated lncRNA 1 (9) and lncRNA growth arrest-specific 5 (GAS5) (10).

Autophagy has recently been indicated to be involved in the regulation of immunity and inflammation. Therefore, the association between autophagy and rheumatic diseases characterized by immune system dysfunction has gained increasing attention from researchers. It has been confirmed that autophagy is involved in the progression of various rheumatic diseases, including systemic lupus erythematosus (SLE), rheumatoid arthritis (RA), osteoarthritis (OA) and systemic sclerosis (11). Furthermore, studies have indicated that autophagy may be involved in the pathogenesis of AS (12-14).

Autophagy regulation is associated with a variety of autophagy genes and lncRNA. Microtubule-associated protein light chain 3 (LC3) is an important gene that regulates autophagy and has been widely used as an autophagosomal marker (15). Beclin1 is important for the recruitment of autophagy proteins to the pre-autophagosome structure and together with vacuolar protein sorting-34 (Vps34), Vps15 and ATG14 form the Beclin1-Vps34-Vps15-ATG14 complex, it triggers vesicle nucleation (16). ATG3 and ATG12-ATG5-ATG16L1 complexes are involved in the expansion and closure of autophagosome membranes as E2 and E3 enzymes in the ATG8 lipidation cascade, respectively (17). It was previously indicated that lncRNA GAS5 is involved in the pathogenesis of immune-associated diseases (18). In the present study, the expression of LC3, Beclin1, ATG3, ATG5, ATG12, ATG16L1 and lncRNA GAS5 in peripheral blood mononuclear cells (PBMCs) from patients with AS and healthy control (HC) subjects was examined, and it was assessed whether the expression of genes associated with autophagy is correlated with clinical parameters and inflammatory cytokines in patients with AS to explore the role and clinical significance of autophagy in the development of AS.

\section{Materials and methods}

Patients and sample collection. Between January 2018 and January 2019, 60 patients with AS were enrolled from the outpatient department and inpatient department of Rheumatology and Immunology of the Affiliated Hospital of North Sichuan Medical College (Nanchong, China). All patients with AS enrolled in this study fulfilled the 1984 modified New York criteria for AS (19). All patients completed a questionnaire, which was used to define the age, sex, and disease severity indexes, including the Bath AS Disease Activity Index (BASDAI) and the Bath AS Functional Index
(BASFI) (20). Laboratory parameters, including the erythrocyte sedimentation rate (ESR) and hypersensitive C-reactive protein (hsCRP), were also recorded. The BASDAI, ESR and hsCRP were used to evaluate disease activity and the normal reference range in the peripheral blood was as follows: ESR, 0-22 mm/1 h; and hsCRP, 0-9 mg/l. The 60 patients were divided into an active disease group $(n=30$; BASDAI $\geq 6$, or $6>\mathrm{BASDAI}>4$ and $\mathrm{ESR}>22 \mathrm{~mm} / 1 \mathrm{~h}$, or $6>\mathrm{BASDAI}>4$ and $\mathrm{hsCRP}>9 \mathrm{mg} / \mathrm{l})$ and an inactive disease group $(\mathrm{n}=30$; BASDAI $\leq 4)$. Control blood samples were obtained from 30 age- and sex-matched healthy subjects who are healthy examinees at the Medical Examination Center of the Affiliated Hospital of North Sichuan Medical College between January 2018 and January 2019 without any evidence of disease. The present study was approved by the ethics committee of the Affiliated Hospital of North Sichuan Medical College (Nanchong, China) and written informed consent was obtained from all of the study subjects enrolled.

$R N A$ extraction and reverse transcription-quantitative PCR $(R T-q P C R)$. The PBMCs were isolated from the blood samples of 30 patients with active AS (AAS) and 30 patients with inactive AS (IAS) and $30 \mathrm{HCs}$ by Ficoll-Hypaque density gradient centrifugation. Total RNA was extracted from PBMCs using TRIzol $^{\circledR}$ reagent (Invitrogen; Thermo Fisher Scientific, Inc.). RT was performed using a PrimeScript RT reagent with gDNA Eraser kit (Takara Bio, Inc.). The synthesized complementary (c)DNA was cryopreserved at $-80^{\circ} \mathrm{C}$ until qPCR was performed. The expression of LC3, Beclin1, ATG5, ATG12, ATG16L1 and lncRNA GAS5 was measured by real-time qPCR using the Applied Biosystems QuantStudio ${ }^{\text {TM }}$ 12K Flex Real-Time PCR system (Applied Biosystems; Thermo Fisher Scientific, Inc.). The reaction mixture contained SYBR Green real-time PCR master mix (Takara Bio, Inc.) $(5.2 \mu \mathrm{l})$, forward and reverse primers $(0.1 \mu \mathrm{l}$ each), cDNA sample $(1 \mu \mathrm{l})$ and double-distilled $\mathrm{H}_{2} \mathrm{O}(3.6 \mu \mathrm{l})$. The thermocycling conditions were as follows: $95^{\circ} \mathrm{C}$ for $10 \mathrm{~min}$, followed by 40 cycles of denaturation at $95^{\circ} \mathrm{C}$ for $10 \mathrm{sec}$ and annealing/extension at $60^{\circ} \mathrm{C}$ for $1 \mathrm{~min}$. All reactions were performed in triplicate. The housekeeping gene $\beta$-actin was used as an internal control. The following primer pairs were used for the qPCR: LC3 forward (F), 5'-AACATG AGCGAGTTGGTCAAG-3' and reverse (R), 5'-GCTCGT AGATGTCCGCGAT-3'; Beclin-1 F, 5'-ACCTCAGCCGAA GACTGAAG-3' and R, 5'-AACAGCGTTTGTAGTTCTGAC A-3'; ATG3 F, 5'-GATGGCGGATGGGTAGATACA-3' and R, 5'-TCTTCACATAGTGCTGAGCAATC-3'; ATG5 F, 5'-CAC TTTGTCAGTTACCAACGTCA-3' and R, 5'-TAGAGCGAA CACGAACCATCC-3'; ATG12 F, 5'-TAGAGCGAACACGAA CCATCC-3' and R, 5'-CACTGCCAAAACACTCATAGA GA-3'; ATG16L1 F, 5'-TAGAGCGAACACGAACCATCC-3' and R, 5'-CCTTTCTGGGTTTAAGTCCAGG-3'; GAS5 F, 5'-AGCAAGCCTAACTCAAGCCATTGG-3' and R, 5'-ACA GTGTAGTCAAGCCGACTCTCC-3' and $\beta$-actin F, 5'-GAG CTACGAGCTGCCTGACG-3' and R, 5'-GTAGTTTCGTGG ATGCCACAG-3'. Relative quantification with the $2^{-\Delta \Delta C q}$ method (21) was used to evaluate the expression of target genes.

Statistical analysis. Statistical analysis was performed using SPSS 22.0 statistical analysis software (IBM Corp.). Quantitative data approximating a normal distribution are 
Table I. Clinical characteristics of patients with AS and HCs.

\begin{tabular}{lcccc}
\hline Characteristics & AS $(\mathrm{n}=60)$ & AAS $(\mathrm{n}=30)$ & IAS $(\mathrm{n}=30)$ & HC $(\mathrm{n}=30)$ \\
\hline Age (years) & $37.1 \pm 9.74$ & $37.67 \pm 10.01$ & $32.67 \pm 7.05$ & $36.53 \pm 9.59$ \\
Sex (male/female) & $48 / 12$ & $26 / 4$ & $22 / 8$ & $23 / 7$ \\
Disease duration (months) & $93.17 \pm 72.61$ & $108.37 \pm 74.60$ & $77.96 \pm 68.43$ & - \\
Positive HLA-B27 & $55(91.7)$ & $27(90)$ & $28(93.3)$ & - \\
BASDAI & $3.98 \pm 1.94$ & $5.58 \pm 0.94^{\mathrm{a}}$ & $2.38 \pm 1.20$ & - \\
BASFI & $2.44 \pm 2.45$ & $3.95 \pm 2.50^{\mathrm{a}}$ & $0.93 \pm 1.12$ & - \\
Anti-TNF inhibitor & $3(5.0 \%)$ & $0(0.0 \%)$ & $3(10.0 \%)$ & - \\
NSAIDs & $15(25.0 \%)$ & $8(26.7 \%)$ & $7(23.3 \%)$ & - \\
DMARDs & $18(20.0 \%)$ & $9(30.0 \%)$ & $9(30.0 \%)$ & - \\
\hline
\end{tabular}

${ }^{a} \mathrm{P}<0.05$ compared with IAS. Values are expressed as the mean \pm standard deviation or $\mathrm{n}(\%)$. AAS, active ankylosing spondylitis; IAS, inactive ankylosing spondylitis; HC, healthy controls; BASDAI, Bath AS Disease Activity Index; BASFI, Bath AS Functional Index; NSAIDs, non-steroidal anti-inflammatory drugs; HLA, human leukocyte antigen; DMARDs, disease-modifying antirheumatic drugs.

expressed as the mean \pm standard deviation. Statistical analysis of demographics, clinical and laboratory indicators were compared using independent samples t-test, Mann-Whitney $\mathrm{U}$ test and one-way ANOVA followed by the LSD post hoc test. Correlations were calculated using Spearman's rank correlation test. A receiver operating characteristic (ROC) curve was drawn and the area under the ROC curve (AUC) was determined to estimate the diagnostic value of lncRNA GAS5 for AS. $\mathrm{P}<0.05$ was considered to indicate statistical significance.

\section{Results}

Baseline characteristics. Table I presents the demographic and clinical characteristics of the 30 patients with AAS, 30 patients with IAS and $30 \mathrm{HCs}$ in the present study. Patients with AS and HCs were matched in terms of age and sex. There were significant differences in the BASDAI and BASFI between the AAS group and the IAS group.

mRNA levels of genes associated with autophagy and lncRNA GAS5 in patients with AS and in HCs. The mRNA levels of LC3, Beclin1, ATG3, ATG5, ATG12, ATG16L1 and the levels of 1ncRNA GAS5 in PBMCs were detected in all samples by RT-qPCR. As presented in Fig. 1A, the expression levels of LC3, ATG5, ATG12, ATG16L1 and lncRNA GAS5 in PBMCs were significantly decreased in the AS compared with the HC group ( $\mathrm{P}<0.05$ for each; Fig. 1A). The expression levels of Beclin1 and ATG3 mRNA in PBMCs was not significantly different between the two groups ( $\mathrm{P}>0.05$ for each; Fig. 1A). Compared with those in the IAS group, the expression levels of ATG5 and ATG12 mRNA were significantly decreased in the AAS group $(\mathrm{P}<0.05$ for each; Fig. 1B). Although no statistical significance was obtained, the expression levels of LC3, Beclin1, ATG3, ATG16L1 and lncRNA GAS5 in patients with AAS were lower than those in patients with IAS ( $P>0.05$ for each; Fig. 1B).

Positive rate of genes associated with autophagy and lncRNA GAS5 in patients with AAS and IAS. The positive rate refers to the proportion of gene expression of patients with AS in the active and inactive groups that increased or decreased relative to the gene expression levels of the HC group (based on the mean of HC). Compared with that in the HC group, the positive rate of LC3 was $80.0 \%$ in the AAS group and $73.3 \%$ in the IAS group. The positive rate of ATG3 was $76.7 \%$ in the IAS group. The positive rate of ATG5 was $100 \%$ in the AAS group and $66.7 \%$ in the IAS group. The positive rate of ATG12 was $100 \%$ in the AAS and $80.0 \%$ in the IAS group. The positive rate of ATG16L1 was $86.7 \%$ in the AAS group. The positive rate of lncRNA GAS5 was $96.7 \%$ in the AAS and $76.7 \%$ in the IAS group (Table II).

Correlation of gene expression with disease activity as well as laboratory indexes in patients with AS. Correlations between detected gene expression levels and clinical parameters reflecting disease activity of AS are presented in Table III. The mRNA expression levels of ATG3, ATG5 and ATG12 exhibited significant negative correlations with the BASDAI $(\mathrm{R}=-0.336, \mathrm{P}<0.01$ for $\mathrm{ATG} 3$; $\mathrm{R}=-0.359, \mathrm{P}<0.01$ for ATG5 and $\mathrm{R}=-0.294, \mathrm{P}<0.05$ for ATG12). The mRNA expression levels of ATG3 were significantly negatively correlated with the BASFI $(\mathrm{R}=-0.360, \mathrm{P}<0.01)$. The mRNA expression levels of ATG3, ATG5, ATG12 and lncRNA GAS5 exhibited significant negative correlations with the ESR $(\mathrm{R}=-0.357, \mathrm{P}<0.01$ for ATG3; $\mathrm{R}=-0.393, \mathrm{P}<0.01$ for ATG5; $\mathrm{R}=-0.313, \mathrm{P}<0.05$ for ATG12 and $\mathrm{R}=-0.424, \mathrm{P}<0.01$ for lncRNA GAS5). The mRNA expression levels of ATG5 and ATG12 were significantly negatively correlated with hsCRP $(\mathrm{R}=-0.328, \mathrm{P}<0.05$ for ATG5; $\mathrm{R}=-0.258, \mathrm{P}<0.05$ for ATG12). The mRNA expression levels of ATG16L1 was significantly negatively correlated with leukocytes $(\mathrm{R}=-0.310, \mathrm{P}<0.05)$. However, the mRNA expression levels of LC3 and Beclin1 were not correlated with any clinical parameters of patients with AS.

Correlations between lncRNA GAS5 and mRNA levels of genes associated with autophagy. Increasing evidence has indicated that lncRNAs are able to regulate autophagy through various mechanisms (7). To assess the potential associations of the levels of lncRNA GAS5 with the mRNA levels of genes associated with autophagy in patients with AS, the correlations 

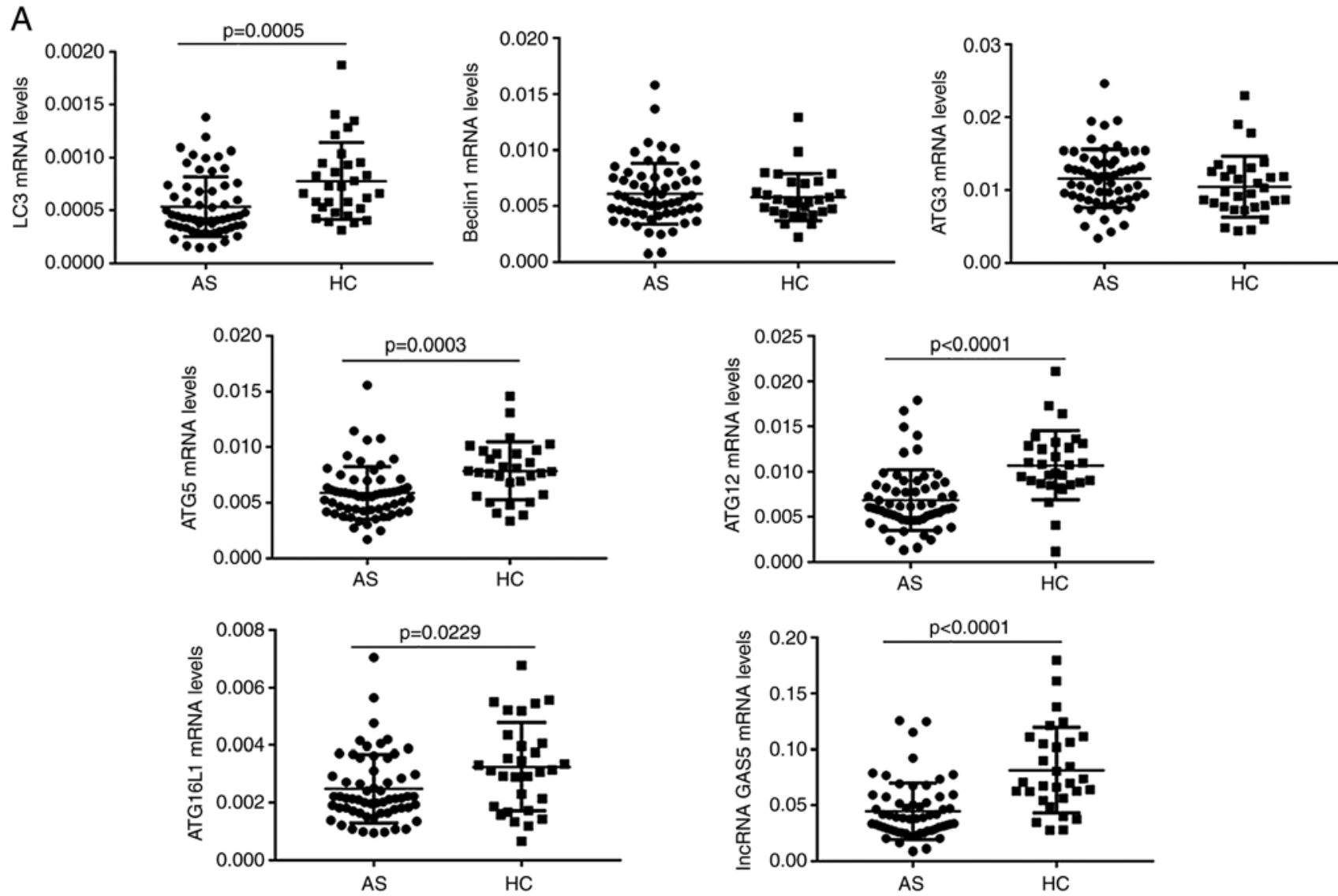

B
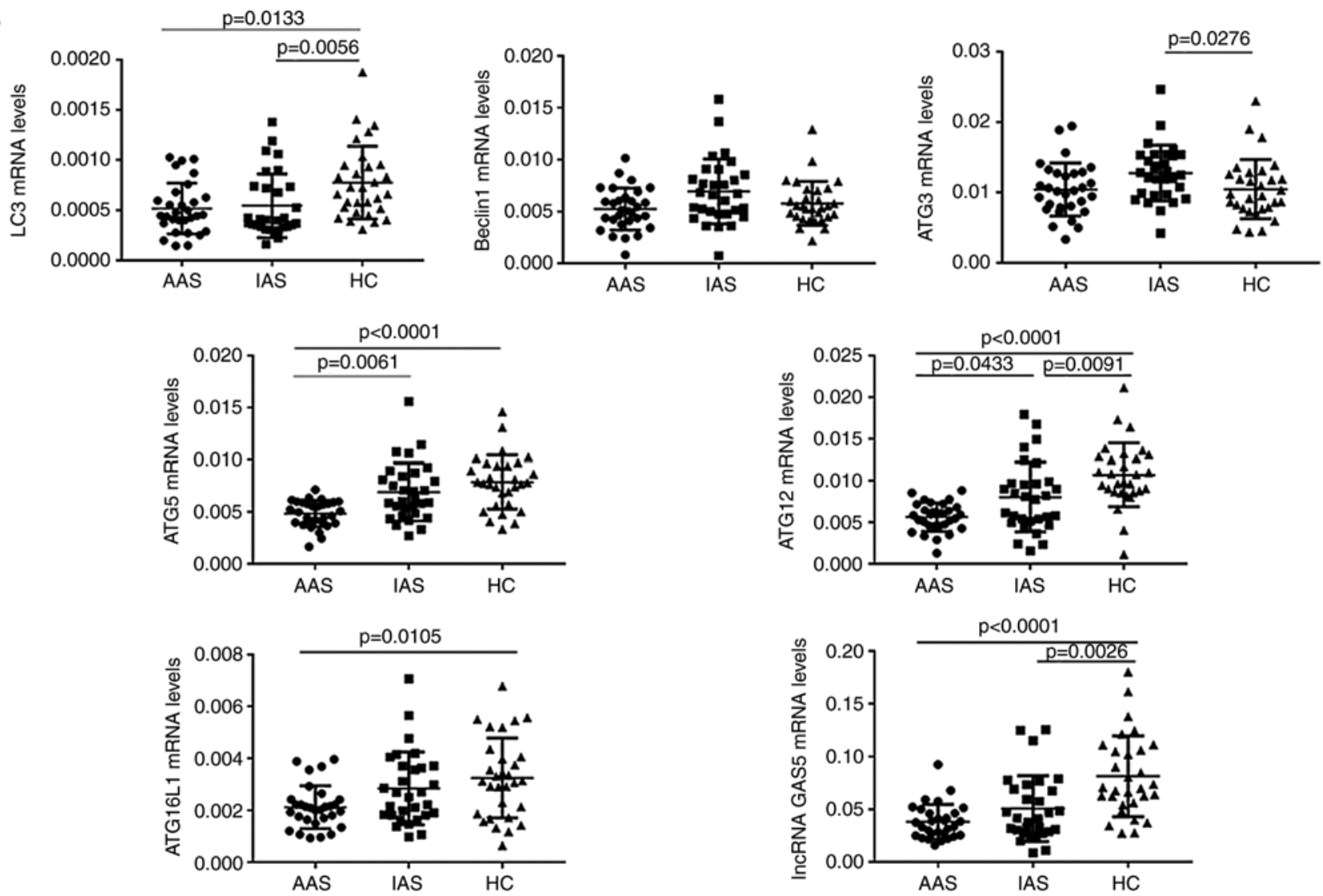

Figure 1. (A) Relative expression of genes associated with autophagy and lncRNA GAS5 in PBMCs of patients with AS ( $\mathrm{n}=60$ ) as compared with HCs $(n=30)$. (B) Expression levels of genes associated with autophagy and lncRNA GAS5 mRNA in PBMCs of patients with AAS ( $n=30)$, IAS ( $n=30)$ and HCs $(n=30)$ were compared. $\mathrm{P}<0.05$ was considered to indicate statistical significance. AAS, active ankylosing spondylitis; IAS, inactive AS; HC, healthy control; ATG16L1, autophagy-related gene 16 ligand 1; LC3, microtubule-associated protein 1 light chain 3; lncRNA, long non-coding RNA; PBMCs, peripheral blood mononuclear cells; GAS5, growth arrest-specific 5 . 
Table II. Rate of positive expression of genes associated with autophagy and lncRNA GAS5 in patients with AAS and IAS (\%).

\begin{tabular}{lccccc}
\hline Group & LC3 & ATG5 & ATG12 & ATG16L1 & IncRNA GAS5 \\
\hline AAS & 83.3 & 100.0 & 100.0 & 86.7 & 96.7 \\
IAS & 80.0 & 66.7 & 80.0 & - & 90 \\
\hline
\end{tabular}

AAS, active ankylosing spondylitis; IAS, inactive ankylosing spondylitis; ATG16L1, autophagy-related gene 16 ligand 1; LC3, microtubule-associated protein 1 light chain 3; lncRNA, long non-coding RNA; GAS5, growth arrest-specific 5; -, the positive rate was not calculated because there was no significant difference in gene expression in the AAS or IAS group compared with the HC group.

between lncRNA GAS5 and LC3, Beclin1, ATG3, ATG5, ATG12 and ATG16L1 were analysed (Fig. 2). The results indicated that the levels of 1ncRNA GAS5 were positively correlated with the levels of Beclin1 $(\mathrm{R}=0.454, \mathrm{P}<0.001$; Fig. 2A), ATG3 ( $R=0.322, P=0.012$; Fig. 2B), ATG5 ( $R=0.500$, $\mathrm{P}<0.001$; Fig. 2C), ATG12 ( $\mathrm{R}=0.317, \mathrm{P}=0.014$; Fig. 2D) and ATG16L1 ( $R=0.279, \mathrm{P}=0.031$; Fig. 2E), respectively. However, no significant correlation was observed between IncRNA GAS5 and $\mathrm{LC} 3$ in patients with AS (R=0.043, $\mathrm{P}=0.741$; Fig. 2F).

Downregulation of IncRNA GAS5 has diagnostic value for AS. An ROC curve was established to determine the diagnostic value of lncRNA GAS5 in PBMCs for AS (Fig. 3). The AUC of the ROC for the use of IncRNA GAS5 to diagnose AS was 0.808 with a 95\% CI of 0.714-0.902 and cut-off value of 0.729 $(\mathrm{P}<0.0001)$.

\section{Discussion}

There is a growing body of evidence suggesting that autophagy is involved in the pathogenesis of AS (12-14). To the best of our knowledge, the present study was the first to determine the expression levels of genes associated with autophagy in different disease activity stages of AS. The key findings of the present study are that ATG5 and ATG12 may serve as novel biomarkers of AS activity and that lncRNA GAS5 is likely involved in the pathogenesis of AS.

In a previous study, Neerinckx et al (12) indicated that the expression levels of ATG16L1 were lower in PBMCs of patients with AS than in healthy controls, whereas ATG5 expression was not significantly different between the two groups. Furthermore, Park et al (13) and Wang et al (14) determined that the expression levels of LC3, Beclin1 and ATG5 mRNA were significantly decreased in PBMCs of patients with AS. In the present study, a decrease in the expression levels of LC3, ATG5, ATG12 and ATG16L1 was observed in patients with AS, whereas the expression levels of Beclin1 and ATG3 were not statistically different between the patients with AS and HCs. The results of the present study are not completely consistent with the results of Neerinckx et al (12), which may be due to differences in populations. Of note, the results for ATG5 in the present study are consistent with the results of Park et al (13) and Wang et al (14), and all of these studies were performed on Asian populations, while the subjects of Neerinckx et al (12) were of Caucasian ethnicity. Furthermore, the expression of Beclin1 in the present study was different from that of Park et al (13). The reason for the inconsistent results may be that although the subjects of the present study were randomly selected, the proportion of AAS and IAS patients is equal, which may lead to selection bias. Different from these previous studies, staging of different disease activities in patients with AS was performed in the present study and the expression levels of genes associated with autophagy were compared between the active and inactive phases. In the present study, the expression levels of ATG5 and ATG12 were significantly lower in patients with AAS than those in patients with IAS. Although no statistical significance was obtained, the expression levels of Beclin1, ATG3 and ATG16L1 in patients with AAS were lower than those in patients with IAS. Therefore, the present data indicated that the level of autophagy in patients with AS was negatively correlated with the severity and activity of the disease. In addition to AS, autophagy dysfunction was also observed in Crohn's disease (22) and Psoriasis (23) as extra-articular manifestations of AS, and other rheumatic immune diseases such as SLE (24). These results are consistent with the notion that defective autophagy may cause inflammation and induce or exacerbate autoimmune disease (25), and further support the results of autophagy dysfunction in AS obtained in the present study.

Autophagy is an important mechanism by which cells decompose cell components through lysosomes or vacuoles to maintain normal physiological activity and homeostasis of the body $(5,6)$. The formation of autophagy is a dynamic process, which may be roughly divided into the following stages: Initiation, nucleation, elongation, fusion and degradation (26). This process is regulated by a unique set of autophagy genes. Loss or abnormal expression of core autophagy genes at various stages of autophagy leads to dysfunctional autophagy, which leads to a variety of diseases. Beclin1 is mainly involved in the initiation stage of autophagy and is an important gene facilitating initiate autophagy. The Atg12-Atg5-Atg16L1 complex is essential for the formation of autophagosomes and contributes to the expansion of the phagophore. ATG3 is involved in the lipidation of LC3 as an E2 enzyme. Furthermore, the ATG12-ATG3 complex has an important role in the basic autophagy flux, endosome function and endolysosomal transport in the late nuclear period (27). A study suggested that neonatal lethality occurred in mice with gene knockout of ATGs involved in the conjugation system (ATG3 ${ }^{-/}$, ATG5 ${ }^{-1}$, $\mathrm{ATG12}^{-/}$and ATG16L11 ${ }^{-/}$) and Beclin1 ${ }^{-/}$mice died of E8.5 (28). LC3-II is located in the outer and inner membranes of autophagosomes and the amount of LC3-II is also widely used for the quantification of autophagic activity (15). In the present study, 
no significant changes in Beclin1 expression in PBMCs from patients with AS were observed, indicating that autophagy initiation may not be impaired. However, the expression of LC3, ATG5, ATG12 and ATG16L1 mRNA in PBMCs of patients with AS was downregulated. These results suggest that there may be certain possible factors in PBMCs of patients with AS: Obstruction of double-membrane autophagosomes, decreased autophagy activity, and impaired accumulation and endolysosomal transport in the late nuclear weeks.

The detailed mechanism of autophagy dysfunction in AS remains to be fully elucidated. It may be speculated that autophagy deficiency is involved in the pathogenesis and disease activity of AS through crosstalk with immune inflammation and endoplasmic reticulum (ER) stress.

It is well known that inhibition of autophagy may lead to increased inflammasome activation (29). In addition to directly promoting the formation of inflammatory bodies and eventually leading to the secretion of inflammatory cytokines, including IL-1 $\beta$ and IL-18, defective autophagy may also indirectly promote the secretion of IL-23 and IL-17 (30). In addition to promoting inflammation, autophagic dysfunction also promotes ER stress in the pathogenesis of AS. Impaired autophagy was observed in colitis and neurodegenerative diseases with active ER stress $(31,32)$. ATG16L1 ${ }^{\mathrm{T} 300 \mathrm{~A}}$, causing impaired autophagy, leads to increased ER stress in Paneth cells (33). Suppression of ATG7 resulted in elevated ER stress and restoration of the expression of ATG7 resulted in dampened ER stress in obesity (34). Therefore, autophagy dysfunction may also promote ER stress. It may therefore be speculated that defective autophagy and ER stress may synergistically promote AS development.

Although no consensus for the use of genes associated with autophagy as diagnostic markers for disease has been reached, it has been indicated that overexpression or low expression of certain autophagy genes may be used as biomarkers for the diagnosis and prognosis of certain diseases (35). The BASDAI has been widely used to asses AS activity and the levels of CRP and ESR are also used to reflect inflammation in patients with AS. In the present study, the expression of ATG5 and ATG12 exhibited a negative correlation with BASDI, ESR and CRP. In addition, the present study determined that ATG5 and ATG12 were downregulated in patients with AAS, as compared with those in patients with IAS. The expression levels of ATG5 and ATG12 in PBMCs of patients with AS were negatively correlated with the severity and activity of the disease, and downregulation of ATG5 and ATG12 may be used as an indicator of the activity of AS.

LncRNA GAS5 may be involved in the development of autoimmune diseases by regulating the proliferation and activity of immune cells and participating in immune responses. Previous studies have indicated that lncRNA GAS5 is abnormally expressed in various immune-associated diseases, including RA, SLE and multiple sclerosis (18). In the present study, lncRNA GAS5 was downregulated in patients with AS compared with that in HCs. It was therefore indicated that IncRNA GAS5 may be a suppressor for AS. In addition, studies have found that the expression of 1ncRNA GAS5 is reduced in most tumour types (36), including prostate cancer, renal cell carcinoma, ovarian cancer, cervical cancer and osteosarcoma. Of note, the occurrence of tumours is associated 

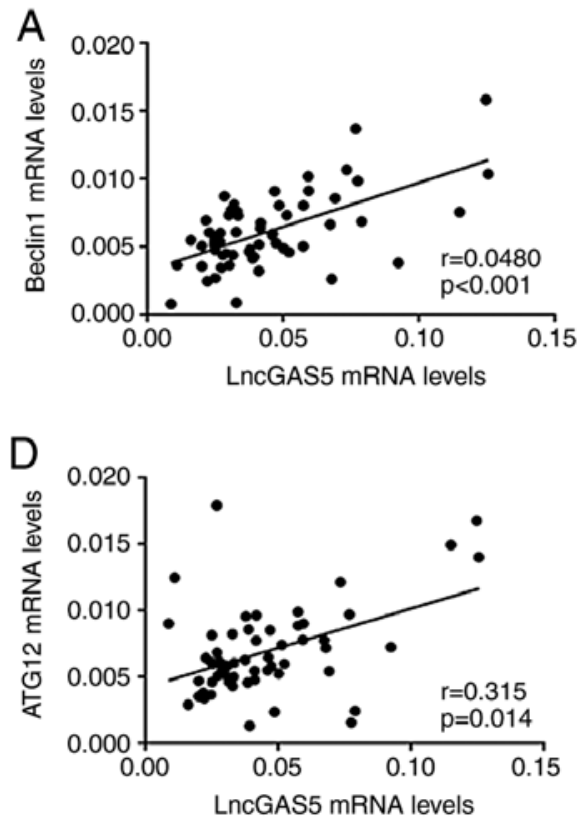

B

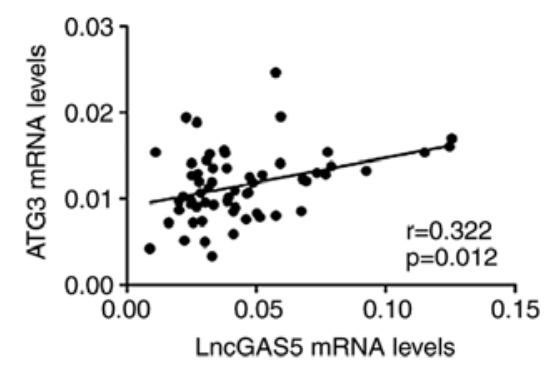

E

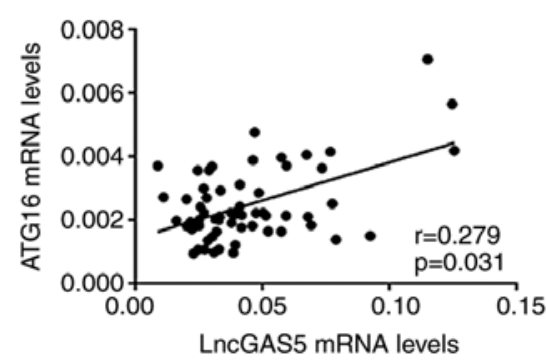

C

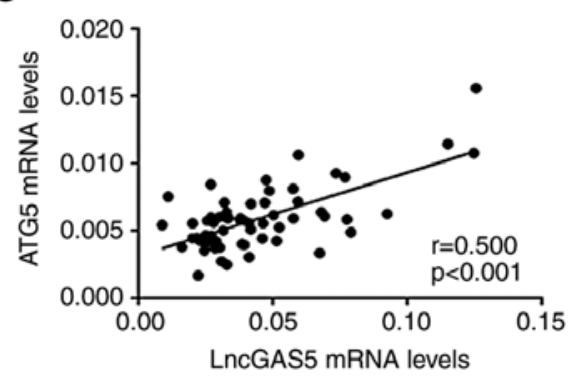

$\mathrm{F}$

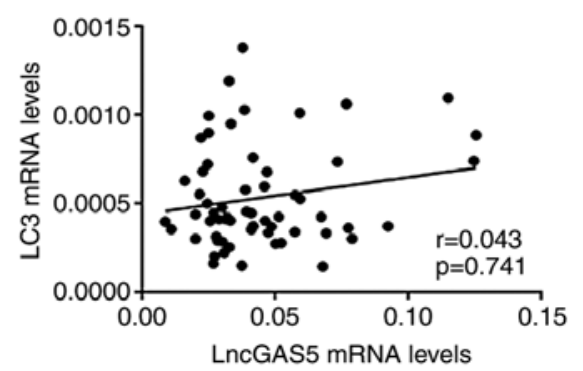

Figure 2. Correlations between lncRNA GAS5 levels and the mRNA levels of genes associated with autophagy in the PBMCs of patients with AS. (A-E) The expression levels of 1ncRNA GAS5 mRNA were positively correlated with (A) Beclin1, (B) ATG3, (C) ATG5, (D) ATG12 and (E) ATG16, but (F) there was no significant correlation with LC3. The correlations were evaluated with Spearman's non-parametric test. P<0.05 was considered to indicate a significant difference. ATG16L1, autophagy-related gene 16 ligand 1; LC3, microtubule-associated protein 1 light chain 3; lncRNA, long non-coding RNA; GAS5, growth arrest-specific 5 .

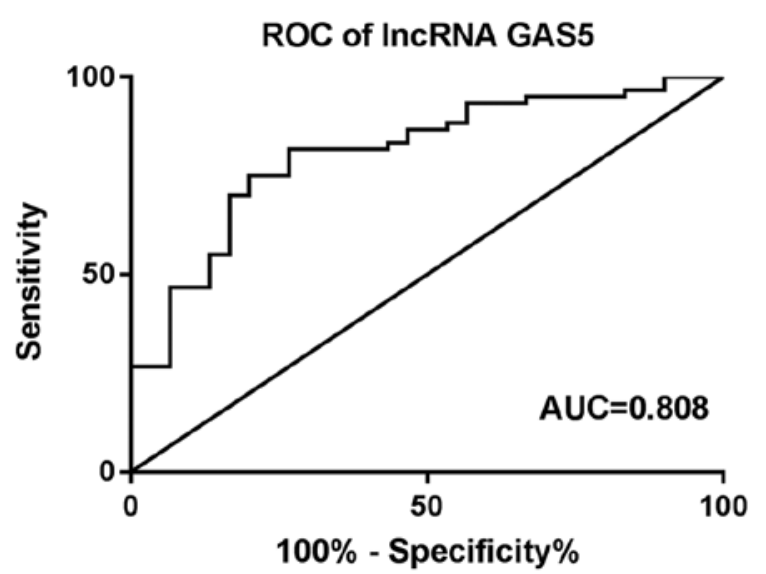

Figure 3. ROC curve for analyzing the diagnostic value of lncRNA GAS5 for ankylosing spondylitis. ROC, receiver operating characteristic; lncRNA, long non-coding RNA; GAS5, growth arrest-specific 5.

with the immune inflammatory microenvironment, mainly manifested as chronic inflammation (37). Therefore, it further supports that lncRNA GAS5 may be involved in the regulation of the pathogenesis of diseases related to immune dysfunction. However, the detailed role and mechanism of 1ncRNA GAS5 still needs to be further clarified. Evidence has indicated that lncRNA GAS5 promoted autophagy through interactions with the PI3K/AKT/mTOR pathway $(10,38)$. Knockdown of lncRNA GAS5 significantly increased the expression of mTOR and suppressed the expression of ATGs (38). The present study suggested that lncRNA GAS5 was downregulated in PBMCs of patients with AS and was positively correlated with the expression of Beclin1, ATG3, ATG5, ATG12 and ATG16. Thus, these data strongly supported the present hypothesis that IncRNA GAS5 participates in the pathogenesis of AS by regulating autophagy. The precise regulatory mechanism of lncRNA GAS5 in autophagy modulation and its detailed roles in AS still requires further elucidation. The present study preliminarily proved the correlation between lncRNA GAS5 and autophagy genes. In future studies, loss- and gain-offunction studies on lncRNA GAS5 will be performed through gene transfection and other techniques to observe the resulting changes of expression levels of autophagy genes or proteins.

Early diagnosis is key for curing AS. Most patients with AS have a long-term delay in diagnosis (39). Of note, there is currently no gold standard for diagnosis, and thus, it is important to identify diagnostic markers for AS. Although HLA-B27 is closely associated with the pathogenesis of AS and the HLA-B27-positive rate of patients with AS reaches 85-90\% (40), the expression of HLA-B27 may not necessarily be positive in all patients with AS and may also be negative. Therefore, it is necessary to further study biomarkers for AS. The present study indicated that the occurrence and development of AS are associated with changes in multiple lncRNA expression patterns, and certain lncRNAs may become potential biomarkers for the diagnosis of AS (41). LncRNA GAS5 is involved in the occurrence and development of various diseases by regulating various physiological processes, including the cell cycle, growth and apoptosis. Studies have indicated that lncRNA GAS5 is involved in the development of atherosclerosis and associated diseases. Low expression of lncRNA GAS5 may be used as a promising biomarker for the diagnosis of coronary artery disease (42). In the present study, the expression levels of lncRNA GAS5 in patients with AS were significantly lower than those of $\mathrm{HC}$, but there was no significant difference between AAS and IAS. Furthermore, the ROC curve analysis indicated that downregulation of lncRNA 
GAS5 in PBMCs may be used to effectively distinguish patients with AS from healthy individuals. Therefore, lncRNA GAS5 may be an independent diagnostic indicator for AS.

Most diagnostic indicators available are elevated, but a small number of diagnostic indicators are reduced. The decrease of an index in a disease may lead to the occurrence of the disease due to the lack of the index. For instance, studies have indicated that the downregulation of IncRNA-D16366 is a biomarker for the diagnosis of hepatocellular carcinoma (43) and the downregulation of IncRNA activated by TGF- $\beta$ in serum is a reliable diagnostic marker for OA (44). In the present study, the diagnostic value of LncRNA GAS5 was determined by ROC curve analysis. ROC analysis is a method that reflects the sensitivity and specificity of diagnostic indicators for a disease, with sensitivity\% displayed on the ordinate and (100\%-specificity\%) on the abscissa. The AUC indicates the diagnostic efficacy of the indicator. The value of the AUC ranges from 1.0 to 0.5 , with a value closer to 1 indicating better diagnostic efficacy. The results obtained in the present ROC curve analysis is only preliminary. Future studies will further verify the diagnostic efficacy by expanding the sample size, functional experiments and disease controls such as increasing sample size of the rheumatoid arthritis group and osteoarthritis group.

In conclusion, autophagy genes and lncRNA GAS5 were downregulated in AS and the level of autophagy in patients with AS was negatively correlated with the severity and activity of the disease. Furthermore, ATG5 and ATG12 may serve as novel biomarkers of AS activity. LncRNA GAS5 may be an independent diagnostic indicator of AS. These results suggest that autophagy deficiency and the downregulation of lncRNA GAS5 may be associated with the pathogenesis of AS. The downregulated autophagy genes and lncRNA GAS5 may be of potential use for the clinical diagnosis and treatment of AS.

\section{Acknowledgements}

Not applicable.

\section{Funding}

The present study was supported by the Sichuan Youth Science and Technology Innovation Team fund of the Sichuan Provincial Department of Education (grant no. 14TD0021).

\section{Availability of data and materials}

The datasets used and/or analyzed during the current study are available from the corresponding author on reasonable request.

\section{Authors' contributions}

QZ and YQ as the corresponding authors conceived the research design and revised the writing of the manuscript. MT collected of case specimens, performed experiments and drafted the manuscript. TL assisted in the completion of the experiments. YY helped with the collection of patient data and samples, and the completion of certain experiments. JZ purchased reagents and assisted in the completion of preliminary experiments. QX and WZ helped collect specimens. All authors read and approved the final version of the manuscript.

\section{Ethics approval and consent to participate}

This study was approved by the ethics committee of the Affiliated Hospital of North Sichuan Medical College and informed consent from all participants was obtained.

\section{Patient consent for publication}

Not applicable

\section{Competing interests}

The authors declare that they have no competing interests.

\section{References}

1. Wang R and Ward MM: Epidemiology of axial spondyloarthritis: An update. Curr Opin Rheumatol 30: 137-143, 2018.

2. Sieper J and Poddubnyy D: Axial spondyloarthritis. Lancet 390: 73-84, 2017.

3. Smith JA: Update on ankylosing spondylitis: Current concepts in pathogenesis. Curr Allergy Asthma Rep 15: 489, 2015.

4. Saha S, Panigrahi DP, Patil S and Bhutia SK: Autophagy in health and disease: A comprehensive review. Biomed Pharmacother 104: 485-495, 2018.

5. Feng Y, He D, Yao Z and Klionsky DJ: The machinery of macroautophagy. Cell Res 24: 24-41, 2014.

6. Galluzzi L, Baehrecke EH, Ballabio A, Boya P, Bravo-San Pedro JM, Cecconi F, Choi AM, Chu CT, Codogno P, Colombo MI, et al: Molecular definitions of autophagy and related processes. EMBO J 36: 1811-1836, 2017.

7. Yang L, Wang H, Shen Q, Feng L and Jin H: Long non-coding RNAs involved in autophagy regulation. Cell Death Dis 8: e3073, 2017.

8. Wang K, Liu CY, Zhou LY, Wang JX, Wang M, Zhao B, Zhao WK, Xu SJ, Fan LH, Zhang XJ, et al: APF lncRNA regulates autophagy and myocardial infarction by targeting miR-188-3p. Nat Commun 6: 6779, 2015.

9. Cai Q, Wang S, Jin L, Weng M, Zhou D, Wang J, Tang Z and Quan Z: Long non-coding RNA GBCDRInc1 induces chemoresistance of gallbladder cancer cells by activating autophagy. Mol Cancer 18: 82, 2019.

10. Gu J, Wang Y, Wang X, Zhou D, Wang X, Zhou M and He Z: Effect of the LncRNA GAS5-MiR-23a-ATG3 axis in regulating autophagy in patients with breast cancer. Cell Physiol Biochem 48: 194-207, 2018.

11. Rockel JS and Kapoor M: Autophagy: Controlling cell fate in rheumatic diseases. Nat Rev Rheumatol 12: 517-531, 2016.

12. Neerinckx B, Carter S and Lories R: IL-23 expression and activation of autophagy in synovium and PBMCs of HLA-B27 positive patients with ankylosing spondylitis. Response to: 'Evidence that autophagy, but not the unfolded protein response, regulates the expression of IL-23 in the gut of patients with ankylosing spondylitis and subclinical gut inflammation' by Ciccia et al. Ann Rheum Dis 73: e68, 2014.

13. Park MC, Kim HW, Lee SW, Song JJ and Park YB: Defective autophagy activity and its association with spinal damage in patients with ankylosing spondylitis. Joint Bone Spine 84: 583-587, 2017.

14. Wang Y, Luo J, Wang X, Yang B and Cui L: MicroRNA-199a-5p induced autophagy and inhibits the pathogenesis of ankylosing spondylitis by modulating the mTOR signaling via directly targeting ras homolog enriched in brain (Rheb). Cell Physiol Biochem 42: 2481-2491, 2017.

15. Kabeya Y, Mizushima N, Ueno T, Yamamoto A, Kirisako T, Noda T, Kominami E, Ohsumi Y and Yoshimori T: LC3, a mammalian homologue of yeast Apg8p, is localized in autophagosome membranes after processing. EMBO J 19: 5720-5728, 2000.

16. Menon MB and Dhamija S: Beclin 1 phosphorylation-at the center of autophagy regulation. Front Cell Dev Biol 6: 137, 2018.

17. Nakatogawa H: Two ubiquitin-like conjugation systems that mediate membrane formation during autophagy. Essays Biochem 55: 39-50, 2013. 
18. Mayama T, Marr AK and Kino T: Differential expression of glucocorticoid receptor noncoding RNA repressor Gas5 in autoimmune and inflammatory diseases. Horm Metab Res 48: 550-557, 2016.

19. van der Linden S, Valkenburg HA and Cats A: Evaluation of diagnostic criteria for ankylosing spondylitis. A proposal for modification of the New York criteria. Arthritis Rheum 27: 361-368, 1984.

20. Madsen OR: Stability of fatigue, pain, patient global assessment and the bath ankylosing spondylitis functional index (BASFI) in spondyloarthropathy patients with stable disease according to the bath ankylosing spondylitis disease activity index (BASDAI). Rheumatol Int 38: 425-432, 2018.

21. Livak KJ and Schmittgen TD: Analysis of relative gene expression data using real-time quantitative PCR and the 2(-Delta Delta C(T)) method. Methods 25: 402-408, 2001.

22. McCarroll SA, Huett A, Kuballa P, Chilewski SD, Landry A, Goyette P, Zody MC, Hall JL, Brant SR, Cho JH, et al: Deletion polymorphism upstream of IRGM associated with altered IRGM expression and Crohn's disease. Nat Genet 40: 1107-1112, 2008.

23. Mahil SK, Twelves S, Farkas K, Setta-Kaffetzi N, Burden AD Gach JE, Irvine AD, Képíró L, Mockenhaupt M, Oon HH, et al: AP1S3 mutations cause skin autoinflammation by disrupting keratinocyte autophagy and up-regulating IL-36 production. J Invest Dermatol 136: 2251-2259, 2016.

24. Martinez J, Cunha LD, Park S, Yang M, Lu Q, Orchard R, Li QZ, Yan M, Janke L, Guy C, et al: Noncanonical autophagy inhibits the autoinflammatory, lupus-like response to dying cells. Nature 533: 115-119, 2016.

25. Yang Z, Goronzy JJ and Weyand CM: Autophagy in autoimmune disease. J Mol Med (Berl) 93: 707-717, 2015.

26. Parzych KR and Klionsky DJ: An overview of autophagy: Morphology, mechanism, and regulation. Antioxid Redox Signal 20: 460-473, 2014.

27. Murrow L, Malhotra R and Debnath J: ATG12-ATG3 interacts with Alix to promote basal autophagic flux and late endosome function. Nat Cell Biol 17: 300-310, 2015.

28. Kuma A, Komatsu M and Mizushima N: Autophagy-monitoring and autophagy-deficient mice. Autophagy 13: 1619-1628, 2017.

29. Shi H, Zhang Z, Wang X, Li R, Hou W, Bi W and Zhang X Inhibition of autophagy induces IL- $1 \beta$ release from ARPE-19 cells via ROS mediated NLRP3 inflammasome activation under high glucose stress. Biochem Biophys Res Commun 463: 1071-1076, 2015

30. Peral de Castro C, Jones SA, Ní Cheallaigh C, Hearnden CA, Williams L, Winter J, Lavelle EC, Mills KH and Harris J: Autophagy regulates IL-23 secretion and innate T cell responses through effects on IL-1 secretion. J Immunol 189: 4144-4153, 2012

31. Kökten T, Gibot S, Lepage P, D'Alessio S, Hablot J, Ndiaye NC, Busby-Venner H, Monot C, Garnier B, Moulin D, et al: TREM-1 inhibition restores impaired autophagy activity and reduces colitis in mice. J Crohns Colitis 12: 230-244, 2018
32. Yin Y, Sun G, Li E, Kiselyov K and Sun D: ER stress and impaired autophagy flux in neuronal degeneration and brain injury. Ageing Res Rev 34: 3-14, 2017.

33. Deuring JJ, Fuhler GM, Konstantinov SR, Peppelenbosch MP, Kuipers EJ, de Haar C and van der Woude CJ: Genomic ATG16L1 risk allele-restricted Paneth cell ER stress in quiescent Crohn's disease. Gut 63: 1081-1091, 2014.

34. Yang L, Li P, Fu S, Calay ES and Hotamisligil GS: Defective hepatic autophagy in obesity promotes ER stress and causes insulin resistance. Cell Metab 11: 467-478, 2010.

35. Castellazzi M, Patergnani S, Donadio M, Giorgi G, Bonora M, Bosi C, Brombo G, Pugliatti M, Seripa D, Zuliani G and Pinton P: Autophagy and mitophagy biomarkers are reduced in sera of patients with Alzheimer's disease and mild cognitive impairment. Sci Rep 9: 20009, 2019.

36. Yu Y and Hann SS: Novel tumor suppressor lncRNA growth arrest-specific 5 (GAS5) in human cancer. Onco Targets Ther 12: 8421-8436, 2019.

37. Yang L and Lin PC: Mechanisms that drive inflammatory tumor microenvironment, tumor heterogeneity, and metastatic progression. Semin Cancer Biol 47: 185-195, 2017.

38. Li L, Huang C, He Y, Sang Z, Liu G and Dai H: Knockdown of long non-coding RNA GAS5 increases miR-23a by targeting ATG3 involved in autophagy and cell viability. Cell Physiol Biochem 48: 1723-1734, 2018.

39. Guillemin F, Briancon S, Pourel J and Gaucher A: Long-term disability and prolonged sick leaves as outcome measurements in ankylosing spondylitis. Possible predictive factors. Arthritis Rheum 33: 1001-1006, 1990.

40. Golder V and Schachna L: Ankylosing spondylitis: An update. Aust Fam Physician 42: 780-784, 2013.

41. Xu Z, Zhou X, Li H, Chen Q and Chen G: Identification of the key genes and long noncoding RNAs in ankylosing spondylitis using RNA sequencing. Int J Mol Med 43: 1179-1192, 2019.

42. Yin Q, Wu A and Liu M: Plasma long non-coding RNA (lncRNA) GAS5 is a new biomarker for coronary artery disease. Med Sci Monit 23: 6042-6048, 2017.

43. Chao Y and Zhou D: IncRNA-D16366 is a potential biomarker for diagnosis and prognosis of hepatocellular carcinoma. Med Sci Monit 25: 6581-6586, 2019

44. Dang X, Lian L and Wu D: The diagnostic value and pathogenetic role of lncRNA-ATB in patients with osteoarthritis. Cell Mol Biol Lett 23: 55, 2018.

This work is licensed under a Creative Commons Attribution-NonCommercial-NoDerivatives 4.0 International (CC BY-NC-ND 4.0) License. 